\title{
Progress in nonequilibrium quantum field theory III
}

\author{
Jürgen Berges ${ }^{\mathrm{a}}$ and Szabolcs Borsányi ${ }^{\mathrm{b}}$ \\ a Institute for Nuclear Physics \\ Darmstadt University of Technology \\ Schlossgartenstr. 9, 64289 Darmstadt, Germany \\ ${ }^{\mathrm{b}}$ Institute for Theoretical Physics \\ University of Heidelberg, \\ Philosophenweg 16, 69120 Heidelberg, Germany
}

We review recent developments and open questions for the description of nonequilibrium quantum fields, continuing hep-ph/0302210 and hep-ph/0410330 [1].

\section{Introduction and overview}

Nonequilibrium quantum field theory is a research area showing substantially increased theoretical activity in recent years. High-energy particle physicists as well as cosmologists are working on very similar techniques and sometimes even on the same underlying nonequilibrium phenomena. This extends to other rapidly evolving topics such as nonequilibrium dynamics in laboratory experiments of ultracold quantum gases [2].

Many developments are triggered by high-energy physics related to collision experiments of heavy nuclei ("Little Bang") and early universe cosmology ("Big Bang"). Despite substantial differences, there are some remarkable parallels. An important example concerns the role of nonequilibrium instabilities for the process of thermalization. Though the origin of these instabilities can be very different, they always lead to substantial growth of occupation numbers in long wavelength modes on time scales much shorter than the asymptotic thermal equilibration time. For an anisotropic QCD plasma Weibel instabilities may operate [3]. For the reheating of the early universe after inflation a tachyonic or parametric resonance instability can lead to an exponential growth of occupation numbers [4]. Characteristic far-from-equilibrium phenomena, such as an early prethermalization of the equation of state, have been quantitatively studied in that context [ [5, 6]. This is followed by a comparably long quasi-stationary period in a manner reminiscent of Kolmogorov wave turbulence [17, 8]. The turbulent behavior is described by stationary solutions of classical statistical field theory. Quantum corrections finally lead to deviations and thermalization to Bose-Einstein or Fermi-Dirac distributions [ [9, 10, 11, 15].

The presence of strong interactions and/or large occupation numbers from nonequilibrium instabilities require nonperturbative approximations. Aspects of systems with high occupation numbers can be nonperturbatively described using classical field theory methods. However, classical Rayleigh-Jeans divergences and the lack of genuine quan- 
tum effects — such as the approach to quantum thermal equilibrium characterized by Bose-Einstein or Fermi-Dirac statistics - limit their use. Gauge-string duality offers a novel possibility for studying dynamical properties of certain strongly interacting gauge theories, which has led to interesting results [13].

Standard nonperturbative approximations such as based on $1 / N$ expansions of the one-particle irreducible (1PI) effective action can be secular in time and do not provide a valid description, similar to perturbation theory. Suitable resummation techniques can be efficiently formulated using the two-particle irreducible (2PI) effective action [ 14. This has led to successful descriptions of far-from-equilibrium dynamics and subsequent thermalization in scalar and fermionic quantum field theories in various dimensions [ 11, 15, 16, 9, 15, 10, 12, 17. They have also been used to compute transport coefficients [ [18] and to determine the range of validity of transport or semi-classical approaches [ 19, 20, 21].

A nonperturbative description of nonequilibrium quantum fields can be based on the $2 \mathrm{PI}$ $1 / N$ expansion beyond leading order (LO), which has been worked out in detail [ 16, 22, 9, [5, 10, 12, 17]. So far, the results from the $2 \mathrm{PI} 1 / N$ expansion to NLO are the only ones that bridge nonequilibrium instabilities at early times and thermal equilibrium at late times. Recently the 2PI $1 / N$ expansion has been pushed forward towards NNLO for a quantum anharmonic oscillator [23]. A series of precision tests has been performed by now [ 24, 25, 10], including the computation of critical exponents near second-order phase transitions [ [26]. The results exhibit some remarkable properties of the $2 \mathrm{PI}-1 / N$ expansion, curing for instance the spurious small- $N$ divergence of the anomalous dimension seen in the standard 1PI $1 / N$ to NLO. Recently, also the question of the formation of topological defects has been investigated in the large- $N$ expansion for $N=1,2$ in one spatial dimension at zero temperature. Since there are no defects in the corresponding quantum theory at zero temperature, the authors drop quantum corrections and introduce a cooling procedure. Defects should then appear as an artefact of the classical approximation, however, the authors see no sign of them. The size of the observed discrepancies are in accordance with earlier comparisons within classical statistical field theory [27]. An important source of quantitative differences arises from the fact that a large- $N$ expansion to NLO in the limit $N \rightarrow 1$ trivially misses $1 / 3$ of the topologically equivalent diagrams starting at NNLO [ 22 .

A nonperturbative large- $N$ expansion of the $2 \mathrm{PI}$ effective action beyond $\mathrm{LO}$, feasible for the (abelian) case of large numbers of flavors, seems not within reach for $S U(N)$ gauge theories relevant for QCD. In this context it remains an important open question to determine the range of validity of $2 \mathrm{PI}$ loop expansions, in particular, in view of a number of remarkable results from related Dyson-Schwinger equations in thermal equilibrium [ 28. It has been an important step that renormalization of 2PI resummed approximations for gauge theories has been worked out [ 29, 30, extending earlier work for scalar [31] and fermionic theories [32].

Complementary to approaches based on analytic approximations, one of the outstanding problems in nonequilibrium quantum field theory are first-principles simulations on a Minkowski space-time lattice. In the following we will describe recent efforts along these lines, which are still in its infancies. 


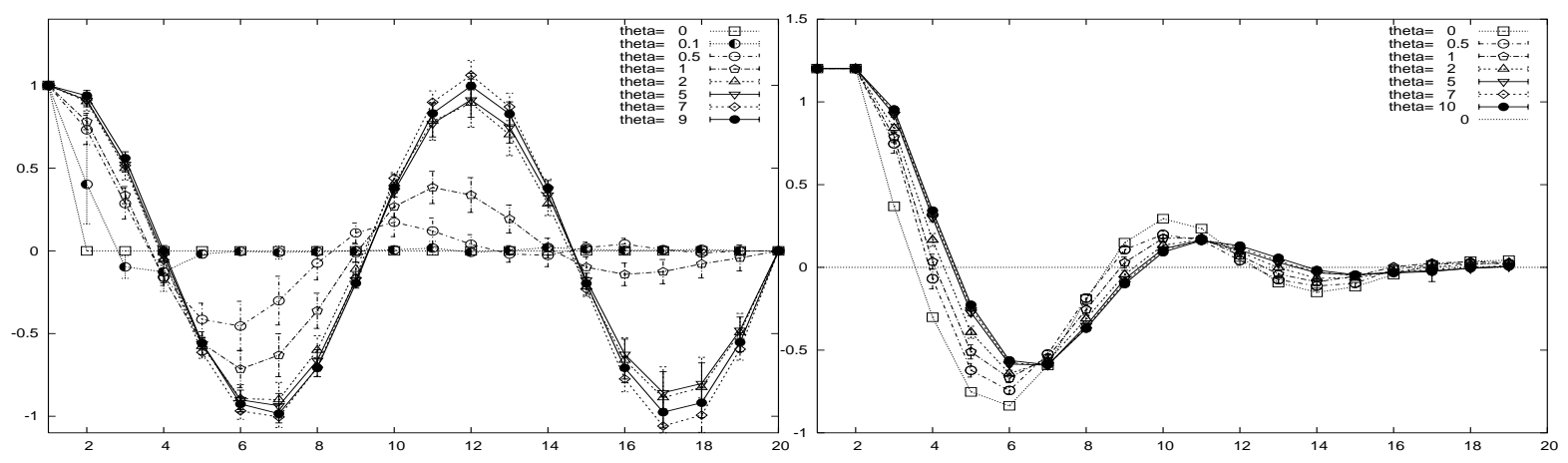

Figure 1. The real part of $\left\langle\int_{x} \varphi(0, \vec{x}) \varphi(t, \vec{x})\right\rangle$ as a funtion of real time $t$ in units of the lattice spacing. Shown are snapshots at different Langevin times $\vartheta$. The LHS corresponds to free field theory, with null starting configuration at $\vartheta=0$. The RHS is for the interacting theory. Here the nonequilibrium classical statistical result is taken as starting configuration, and the Langevin updating for $\vartheta>0$ incorporates quantum corrections [ 37.

\section{Lattice simulations of real-time quantum fields}

Lattice gauge theory calculations are typically based on a Euclidean formulation, where the time variable is analytically continued to imaginary values. By this the quantum theory is mapped onto a statistical mechanics problem, which can be simulated by importance sampling techniques. Recovering real-time properties from the Euclidean formulation is a formidable problem. Direct simulations in Minkowski space-time would be a breakthrough in our efforts to resolve pressing questions, such as early thermalization or the origin of seemingly perfect fluidity in a QCD plasma at RHIC. For real times standard importance sampling is not possible because of a non-positive definite probability measure. Efforts to circumvent this problem include mimicking the real-time dynamics by computer-time evolution in Euclidean lattice simulations [33, 34. A problem in this case is to calibrate the computer time independently of the algorithm. In principle, direct simulations in Minkowski space-time may be obtained using stochastic quantization techniques, which are not based on a probability interpretation [35, 36].

In Ref. [37] this has been recently used to explore nonequilibrium dynamics of an interacting scalar quantum field theory. As an example, Fig. 1 shows the time evolution for the connected part of the unequal-time correlator $\operatorname{Re}\left\langle\int_{x} \varphi(0, \vec{x}) \varphi(t, \vec{x})\right\rangle$, which measures the correlation of the field $\varphi$ at time $t$ with the initial field. Shown are snapshots of the correlator at different Langevin-times. The left figure shows the result for the free field theory with null start configuration. The Langevin updating reproduces the correct freefield result. The right figure gives an example for the interacting theory, which exhibits a finite characteristic damping time. One observes good convergence properties of the quantum simulations, which is a remarkable result. For given initial field configurations at time $t=0$, very different starting configurations for the $(3+1)$-dimensional space-time lattice converge to the same nonequilibrium dynamics for all $t>0$ [37]. 


\subsection{Real-time stochastic quantization}

In real-time stochastic quantization the quantum ensemble is constructed by a stochastic process in an additional "Langevin-time" using the reformulation for the Minkowskian path integral [38, 39]: The quantum fields are defined on a $d$-dimensional physical spacetime lattice, while the updating procedure employs a Langevin equation with a complex driving force in an additional, unphysical "time" direction. Though more or less formal proofs of equivalence of the stochastic approach and the path integral formulation have been given for Minkowski space-time, not much is known about the general convergence properties and its reliability beyond free-field theory or simple toy models [39]. More advanced applications concern simulations in Euclidean space-time with non-real actions [ 40, 41.

Below we follow Ref. [42] and discuss real-time stochastic quantization for a scalar theory and $S U(N)$ pure gauge theory relevant for QCD. For a scalar theory with Minkowski action $S[\varphi]$ the Langevin updating equation reads

$\varphi^{\prime}(x)=\varphi(x)+i \epsilon \frac{\delta S[\varphi]}{\delta \varphi(x)}+\sqrt{\epsilon} \eta(x)$,

with Gaussian noise

$\langle\eta(x)\rangle_{\eta}=0, \quad\langle\eta(x) \eta(y)\rangle_{\eta}=2 \delta(x-y)$.

The sum over all Langevin steps, $\vartheta \equiv \sum \epsilon$, corresponds to Langevin-time.

For $S U(N)$ gauge theory on a $\left(N_{s} a_{s}\right)^{3} \times N_{t} a_{t}$ lattice the real-time classical action reads

$$
\begin{aligned}
S[U]= & -\beta_{0} \sum_{x} \sum_{i}\left\{\frac{1}{2 \operatorname{Tr} \mathbf{1}}\left(\operatorname{Tr} U_{x, 0 i}+\operatorname{Tr} U_{x, 0 i}^{-1}\right)-1\right\} \\
& +\beta_{s} \sum_{x} \sum_{\substack{i, j \\
i<j}}\left\{\frac{1}{2 \operatorname{Tr} \mathbf{1}}\left(\operatorname{Tr} U_{x, i j}+\operatorname{Tr} U_{x, i j}^{-1}\right)-1\right\},
\end{aligned}
$$

with spacial indices $i, j=1,2,3$. It is described in terms of the gauge invariant plaquette variable

$U_{x, \mu \nu} \equiv U_{x, \mu} U_{x+\hat{\mu}, \nu} U_{x+\hat{\nu}, \mu}^{-1} U_{x, \nu}^{-1}$.

Here $U_{x, \mu}$ is the parallel transporter associated with the link from the neighbouring lattice point $x+\hat{\mu}$ to the point $x$ in the direction of the lattice axis $\mu=0,1,2,3$. The couplings are

$\beta_{0} \equiv \frac{2 \gamma \operatorname{Tr} \mathbf{1}}{g_{0}^{2}}, \quad \beta_{s} \equiv \frac{2 \operatorname{Tr} \mathbf{1}}{g_{s}^{2} \gamma}$,

with the anisotropy parameter $\gamma \equiv a_{s} / a_{t}$. The Langevin updating equation for $U_{x, \mu}$ then reads

$U_{x, \mu}^{\prime}=\exp \left\{i \lambda_{a}\left(\epsilon i D_{x \mu a} S[U]+\sqrt{\epsilon} \eta_{x \mu a}\right)\right\} U_{x, \mu}$,

with

$i D_{x \mu a} S[U]=-\frac{1}{2 N} \sum_{\substack{\nu=0 \\ \nu \neq \mu}}^{3} \beta_{\mu \nu} \operatorname{Tr}\left(\lambda_{a} U_{x, \mu} C_{x, \mu \nu}-\bar{C}_{x, \mu \nu} U_{x, \mu}^{-1} \lambda_{a}\right)$. 
For a compact notation we have defined $\beta_{i j} \equiv \beta_{s}$ and $\beta_{0 i} \equiv \beta_{i 0} \equiv-\beta_{0}$ and

$$
\begin{aligned}
& C_{x, \mu \nu}=U_{x+\hat{\mu}, \nu} U_{x+\hat{\nu}, \mu}^{-1} U_{x, \nu}^{-1}+U_{x+\hat{\mu}-\hat{\nu}, \nu}^{-1} U_{x-\hat{\nu}, \mu}^{-1} U_{x-\hat{\nu}, \nu} \\
& \bar{C}_{x, \mu \nu}=U_{x, \nu} U_{x+\hat{\nu}, \mu} U_{x+\hat{\mu}, \nu}^{-1}+U_{x-\hat{\nu}, \nu}^{-1} U_{x-\hat{\nu}, \mu} U_{x+\hat{\mu}-\hat{\nu}, \nu} .
\end{aligned}
$$

It is shown in Ref. [42] (see also Ref. [43]) that stationary solutions of equations (11) or (66) always fulfill the infinite set of (symmetrized) Dyson-Schwinger identities of the respective quantum field theory. For instance, for the scalar theory one finds from the fixed points of the Langevin equation (1D) the infinite hierarchy of identities

$$
\begin{aligned}
& \left\langle\frac{\delta S[\varphi]}{\delta \varphi(x)}\right\rangle_{\eta}=0, \\
& \left\langle\frac{\delta S[\varphi]}{\delta \varphi(x)} \varphi(y)\right\rangle_{\eta}+\left\langle\frac{\delta S[\varphi]}{\delta \varphi(y)} \varphi(x)\right\rangle_{\eta}=2 i \delta(x-y), \\
& \left\langle\frac{\delta S[\varphi]}{\delta \varphi(x)} \varphi(y) \varphi(z)\right\rangle_{\eta}+\left\langle\frac{\delta S[\varphi]}{\delta \varphi(y)} \varphi(x) \varphi(z)\right\rangle_{\eta}+\left\langle\frac{\delta S[\varphi]}{\delta \varphi(z)} \varphi(x) \varphi(y)\right\rangle_{\eta}= \\
& 2 i\left(\langle\varphi(x)\rangle_{\eta} \delta(y-z)+\langle\varphi(y)\rangle_{\eta} \delta(x-z)+\langle\varphi(z)\rangle_{\eta} \delta(x-y)\right),
\end{aligned}
$$

and correspondingly for the higher $n$-point functions in Minkowski space-time. If the Langevin updating converges, which is the case for the implementation of Ref. [42], then it solves the correct infinite set of Dyson-Schwinger identities. In addition, Euclidean theories with real actions can be shown to have a unique solution based on positivity arguments. A similar argument fails for real-time stochastic quantization. In general, here the correct fixed point cannot be chosen a priori without implementing further constraints.

\subsection{Precision tests for scalar and nonabelian gauge theories}

In Ref. [42] tests for real-time stochastic quantization are discussed. Similar to what has been observed in Ref. [37] for scalar fields, also for pure gauge theory one finds that previously reported unstable dynamics represents no problem in practice: A combination of sufficiently small Langevin step size and the use of a "tilted" real-time contour leads to converging results in general. The employed procedure respects gauge invariance and appears to be well under control. This is exemplified for $S U(2)$ gauge theory in $3+1$ dimensions and for a scalar theory in zero spatial dimension, i.e. a quantum anharmonic oscillator. For the latter the Schrödinger equation can be solved as well numerically by diagonalization of the Hamiltonian, for comparison.

For the scalar theory one finds that stochastic quantization accurately describes the time evolution for lattices with sufficiently small real-time extent. This concerns nonequilibrium and equilibrium simulations at weak as well as strong couplings. As an example, Fig. [2] shows results for the time evolution for $\lambda \varphi^{4} / 4$ ! scalar theory in zero spatial dimension. Shown is the two-point correlation function $\langle\varphi(0) \varphi(t)\rangle_{\eta}$ as a function of real time $t$. The simulations are done in thermal equilibrium, in which case the real-time contour extends along the imaginary axis to the inverse temperature $\beta$. For the left figure the real-time extent of the contour is $\Delta t=0.5$. Here the upper branch of the contour has a tilt 

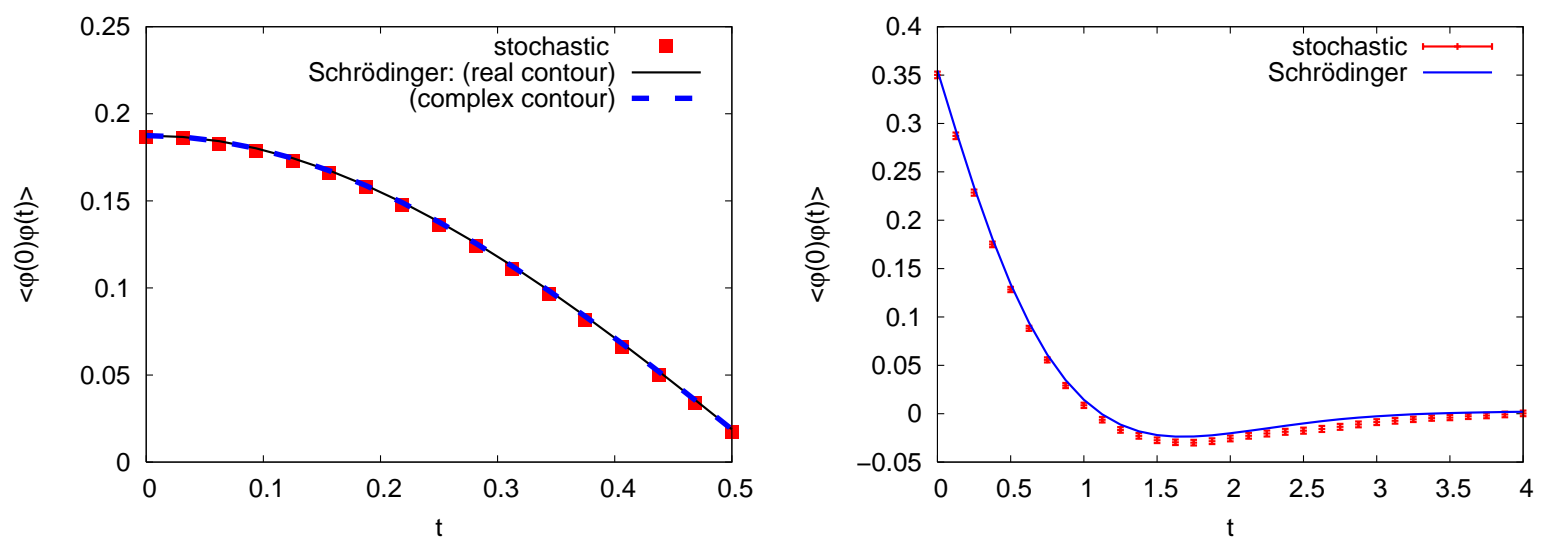

Figure 2. The unequal-time two-point correlation function for the quantum anharmonic oscillator as a function of real time $t$. The (short-time) results obtained from stochastic quantization agree to very good accuracy to those obtained from directly solving the Schrödinger equation. For the left figure a strong coupling $\lambda=96$ and $\beta=1$ is employed, whereas $\lambda=6$ and $\beta=8$ for the right. [42]

of $0.001 \beta$, so it is almost horizontal and, therefore, realizes to high accuracy a real-time contour. For comparison to the stochastic quantization result the time evolution from the Schrödinger equation in Minkowski time as well as using the employed complex times from the corresponding matrix algebra is given. They all agree to very good accuracy.

However, when the real-time extent of the lattice is enlarged the stochastic updating does not converge to the correct solution. Fig. 2 shows the two-point correlation function as a function of real time $t$ for $\lambda=24$ and $\beta=1$. For a short real-time lattice corresponding to $t_{\text {final }}=0.8$ one observes from the left figure excellent agreement of stochastic quantization and Schrödinger equation results. However, doubling the extent of the contour leads to a qualitatively different behavior as given in the right plot of Fig. 2. This difference persists also on finer real-time grids. The non-vanishing imaginary part of the equal-time correlator and the loss of time-translation invariance reflects a non-unitary time evolution.

Doing the equivalent investigation for $S U(2)$ pure gauge theory for thermal equilibrium, one finds a similar behavior with an important difference. The left of Fig. 4 shows the Langevin-time evolution of the spatial plaquette average. This quantity is computed for different complex contours, with the approximation that $g_{0}=g_{s}$. The solid line shows the result for vanishing real-time extent, i.e. for a Euclidean contour corresponding to an inverse temperature $\beta=4$. The different dashed curves correspond to results for complex contours on isosceles triangles each having a different tilt $\alpha$ with respect to the real-time axis. Here $\alpha=0$ would correspond to an infinite extent along the real axis. One observes that with increased real-time extent or smaller tilt $\alpha$ the correct thermal solution is approached less accurately. In particular, it is only approached at intermediate Langevin-times, irrespective of the details of the non-vanishing real-part of the contour. This aspect differs from the scalar case where short real-time extents lead to stable thermal 

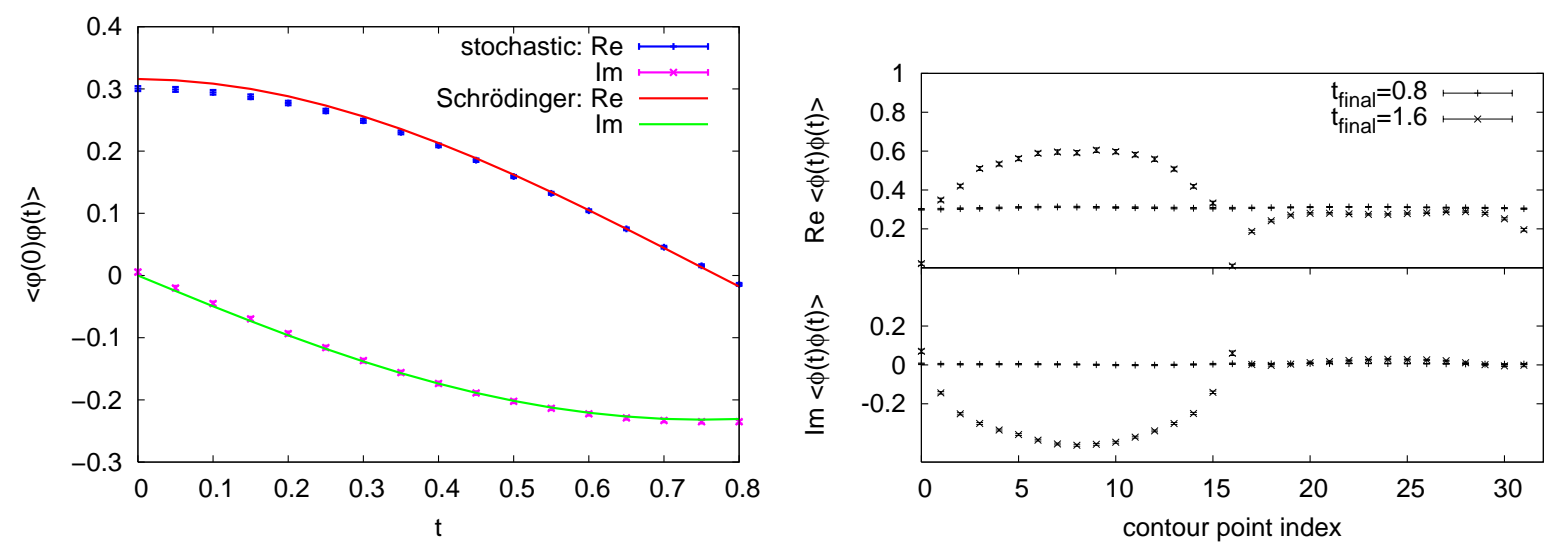

Figure 3. Real and imaginary part of the two-point correlation function for the quantum anharmonic oscillator as a function of real time $t$. Left: for short real-time extent of the lattice the stochastic quantization results agree well to those obtained from the Schrödinger equation. Right: Equal-time correlator as a function of time (index of lattice site along the time-contour). Compared are two simulations where the real-time extent of the lattice differs by a factor of two. The larger lattice leads to a qualitatively different, non-unitary behavior. [42]

solutions.

As mentioned above, all converging solutions of the stochastic dynamics method fulfill the same infinite set of (symmetrized) Dyson-Schwinger identities of the quantum field theory. This is remarkable in view of the different "physical" and "unphysical" solutions that are observed. In Fig. [5 this is visualized for the example of the Dyson-Schwinger equation for a spatial plaquette variable in $S U(2)$ gauge theory. Plotted are separately the LHS and the RHS of the Dyson-Schwinger equation displayed graphically on the right of Fig. 5. The left plot displays the respective LHS and RHS as a function of Langevin-time for a typical contour with tilt $0<\alpha<\pi / 2$. The flow with Langevin-time quickly leads to a rather accurate agreement of both sides such that the Dyson-Schwinger equation is fulfilled. However, after some Langevin-time they start deviating again, finally leading to another stationary value where the LHS and RHS agree to reasonable accuracy.

The evolution with Langevin-time is governed by fixed points and, for the typical case considered in Fig. 5, the correct thermal fixed point is approached at first. However, it is not stable and the Langevin flow exhibits a crossover to another (stable) fixed point.

In conclusion, short real-time physics in thermal equilibrium can be reproduced reasonably well if the length of the real time contour is small on the scale of the inverse temperature $\beta$. For longer contours the boundary conditions in physical time do not seem to constrain enough the Langevin flow and the 'life-time' of the thermal (physical) fixed point decreases (nonabelian gauge theory), or the fixed point becomes fully unstable (scalar theory). A second, apparently stable fixed point develops for large Langevin times. This latter represents an unphysical, non-unitary regime, to be recognized by nontranslational invariant expectation functions and violation of the unsymmetrized Dyson- 

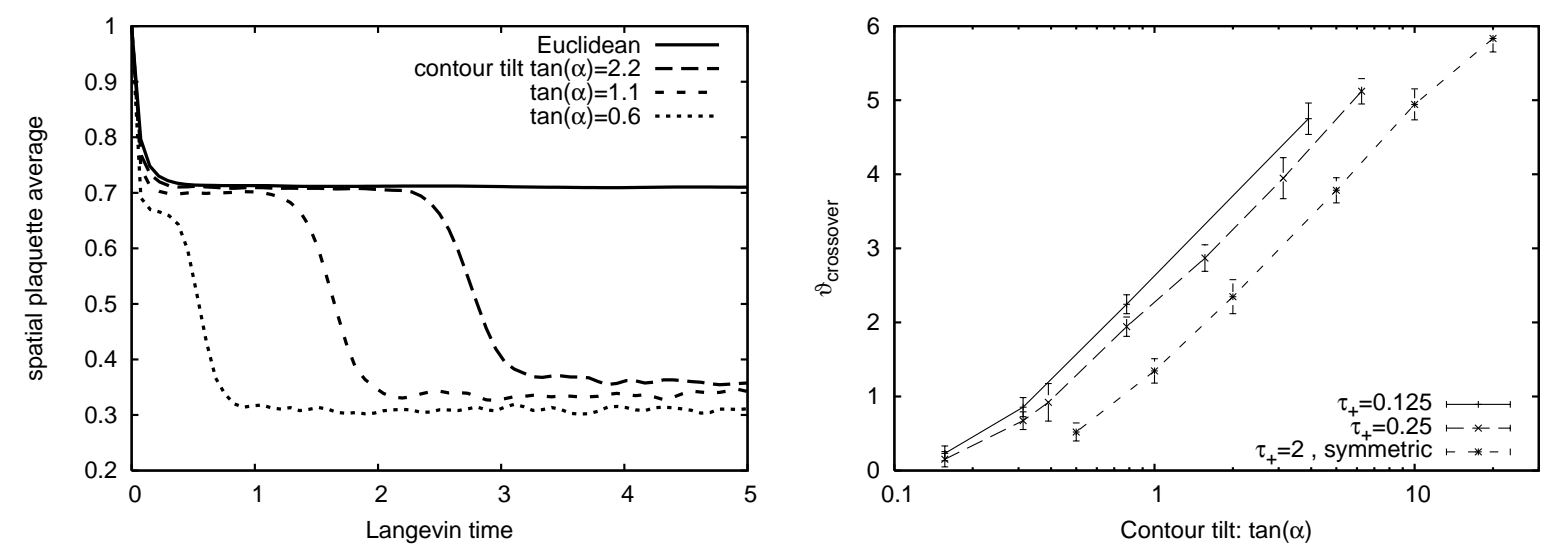

Figure 4. The spatial plaquette average for $S U(2)$ gauge theory in thermal equilibrium as a function of Langevin time. Shown are results for different complex contours. Here $\alpha=0$ corresponds to a contour with an infinite extent along the real-time axis, while $\alpha=\pi / 2$ denotes the Euclidean contour. The longer the real-time component of the contour the less accurate the thermal solution is approached. Here $\vartheta_{\text {crossover }}$ denotes the Langevin-time at which the crossover to the non-unitary fixed point occurs. [42]

Schwinger identities (while the symmetrized ones are still satisfied, indicating convergence) [42. Further work has to concentrate on further means for controlling and optimizing the method. The method allows for quite some flexibility as to which quantities are chosen to define the stochastic process, or introducing a stochastic re-weighting.

We are indepted to D. Sexty and I-O. Stamatescu for collaboration on this topic.

\section{REFERENCES}

1. J. Berges and J. Serreau in SEWM02, Ed. M. G. Schmidt World Scientific, Singapore, 2003 arXiv:hep-ph/0302210. SEWM04, Eds. K. J. Eskola, K. Kainulainen, K. Kajantie, K. Rummukainen, World Scientific, Singapore, 2005 arXiv:hep-ph/0410330

2. T. Gasenzer, J. Berges, M. G. Schmidt and M. Seco, these proceedings arXiv:cond-mat/0608553.

3. S. Mrowczynski, Phys. Lett. B 314 (1993) 118. M. Strickland, these proceedings arXiv:hep-ph/0608173. G. D. Moore, these proceedings.

4. J. H. Traschen and R. H. Brandenberger, Phys. Rev. D 42 (1990) 2491. L. Kofman, A. D. Linde and A. A. Starobinsky, Phys. Rev. Lett. 73 (1994) 3195. L. Kofman, these proceedings.

5. J. Berges, S. Borsanyi and C. Wetterich, Phys. Rev. Lett. 93 (2004) 142002.

6. D. I. Podolsky, G. N. Felder, L. Kofman and M. Peloso, Phys. Rev. D 73 (2006) 023501. J. F. Dufaux, G. Felder, L. Kofman, M. Peloso and D. Podolsky, JCAP 0607 (2006) 006.

7. R. Micha and I. I. Tkachev, Phys. Rev. Lett. 90 (2003) 121301. Phys. Rev. D 70 (2004) 043538. 

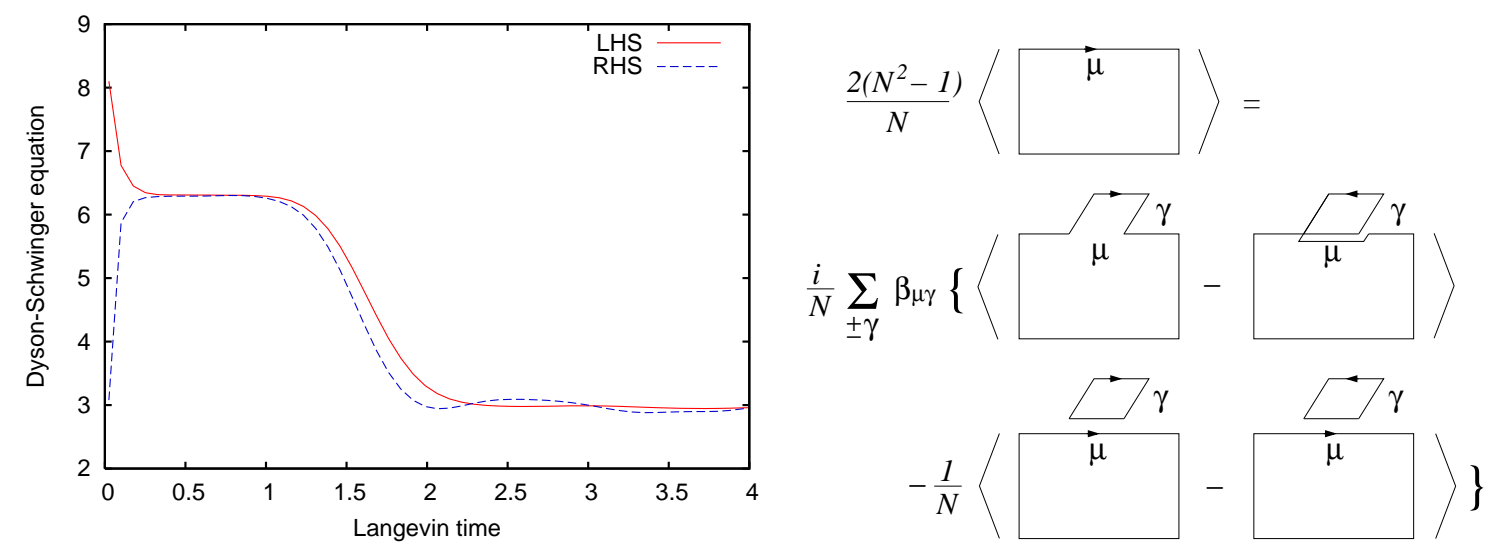

Figure 5. Numerical measurement (left) of the Dyson-Schwinger equation (right) for a spatial plaquette variable. [42]

8. P. Arnold and G. D. Moore, Phys. Rev. D 73 (2006) 025006. Phys. Rev. D 73 (2006) 025013.

9. J. Berges and J. Serreau, Phys. Rev. Lett. 91 (2003) 111601.

10. A. Arrizabalaga, J. Smit and A. Tranberg, JHEP 0410 (2004) 017.

11. J. Berges and J. Cox, Phys. Lett. B 517 (2001) 369. J. Berges, S. Borsanyi and J. Serreau, Nucl. Phys. B 660 (2003) 51.

12. J. Berges, "Introduction to nonequilibrium quantum field theory," AIP Conf. Proc. 739 (2005) 3; arXiv:hep-ph/0409233.

13. G. Policastro, D. T. Son and A. O. Starinets, Phys. Rev. Lett. 87 (2001) 081601. A. O. Starinets, these proceedings.

14. J. M. Cornwall, R. Jackiw and E. Tomboulis, Phys. Rev. D 10 (1974) 2428.

15. G. Aarts, J. Berges, Phys. Rev. D 64 (2001) 105010. F. Cooper, J. F. Dawson and B. Mihaila, Phys. Rev. D 67 (2003) 056003. D.J. Bedingham, Phys. Rev. D 68 (2003) 105007. S. Juchem, W. Cassing, C. Greiner, Phys. Rev. D 69 (2004) 025006. A. Arrizabalaga, J. Smit and A. Tranberg, Phys. Rev. D 72 (2005) 025014.

16. J. Berges, Nucl. Phys. A 699 (2002) 847.

17. T. Gasenzer, J. Berges, M. G. Schmidt and M. Seco, Phys. Rev. A 72 (2005) 063604.

18. G. Aarts and J. M. Martinez Resco, Phys. Rev. D 68 (2003) 085009. JHEP 0402 (2004) 061. arXiv:hep-ph/0402192. JHEP 0503 (2005) 074.

19. J. Berges and S. Borsanyi, Phys. Rev. D 74 (2006) 045022. Nucl. Phys. B 727 (2005) 244.

20. S. Juchem, W. Cassing and C. Greiner, Nucl. Phys. A 743 (2004) 92.

21. M. Lindner and M. M. Muller, Phys. Rev. D 73 (2006) 125002.

22. G. Aarts, D. Ahrensmeier, R. Baier, J. Berges and J. Serreau, Phys. Rev. D 66 (2002) 045008 .

23. G. Aarts and A. Tranberg, Phys. Rev. D 74 (2006) 025004.

24. G. Aarts and J. Berges, Phys. Rev. Lett. 88, 041603 (2002).

25. K. Blagoev, F. Cooper, J. Dawson and B. Mihaila, Phys. Rev. D 64 (2001) 125003.

26. M. Alford, J. Berges and J. M. Cheyne, Phys. Rev. D 70 (2004) 125002. 
27. A. Rajantie and A. Tranberg, arXiv:hep-ph/0607292

28. C. S. Fischer, J. Phys. G 32 (2006) R253.

29. U. Reinosa and J. Serreau, JHEP 0607 (2006) 028; U. Reinosa, these proceedings arXiv:hep-ph/0609058.

30. J. M. Pawlowski, arXiv:hep-th/0512261.

31. H. van Hees, J. Knoll, Phys. Rev. D 65 (2002) 025010; Phys. Rev. D 65 (2002) 105005; Phys. Rev. D 66 (2002) 025028. J.P. Blaizot, E. Iancu, U. Reinosa, Phys. Lett. B 568 (2003) 160; Nucl. Phys. A 736 (2004) 149. J. Berges, S. Borsanyi, U. Reinosa and J. Serreau, Phys. Rev. D 71 (2005) 105004; Annals Phys. 320 (2005) 344. F. Cooper, B. Mihaila and J. F. Dawson, Phys. Rev. D 70 (2004) 105008; Phys. Rev. D 71 (2005) 096003.

32. U. Reinosa, Nucl. Phys. A 772 (2006) 138.

33. J.D. Gunton, M. San Miguel and P. S. Sahni, in "Phase Transitions and Critical Phenomena, v. 8", ed. C. Domb and J. L. Lebowitz, Academic Press (1983).

34. T. R. Miller and M. C. Ogilvie, Phys. Lett. B 488 (2000) 313. A. Bazavov, B. A. Berg and A. Velytsky, Int. J. Mod. Phys. A 20 (2005) 3459; Phys. Rev. D 74, 014501 (2006). E. T. Tomboulis and A. Velytsky, Phys. Rev. D 72, 074509 (2005) arXiv:hep-ph/0507197.

35. G. Parisi and Y.-S. Wu, Sci. Sin. 24 (1981) 483. For reviews see E. Seiler, Schladming (1984) 259. P. H. Damgaard and H. Hüffel, Phys. Rept. 152 (1987) 227.

36. E. Seiler, I. O. Stamatescu and D. Zwanziger, Nucl. Phys. B 239 (1984) 177; Nucl. Phys. B 239 (1984) 201. G. G. Batrouni et al, Phys. Rev. D 32 (1985) 2736.

37. J. Berges and I. O. Stamatescu, Phys. Rev. Lett. 95 (2005) 202003.

38. J. R. Klauder, in "Recent developments in High Energy Physics," ed. H. Mitter and C. B. Lang, Springer (1983); Phys. Rev. A 29 (1984) 2036. G. Parisi, Phys. Lett. B 131 (1983) 393. For a review see K. Okano, L. Schulke and B. Zheng, Prog. Theor. Phys. Suppl. 111 (1993) 313.

39. H. Hüffel and H. Rumpf, Phys. Lett. B 148 (1984) 104. H. Hüffel and P. V. Landshoff, Nucl. Phys. B 260 (1985) 545. E. Gozzi, Phys. Lett. B 150 (1985) 119. D. J. E. Callaway et al, Nucl. Phys. B 262, 19 (1985). H. Nakazato and Y. Yamanaka, Phys. Rev. D 34 (1986) 492. H. Okamoto et al, Nucl. Phys. B 324 (1989) 684.

40. J. Flower, S. W. Otto and S. Callahan, Phys. Rev. D 34 (1986) 598. J. Ambjorn, M. Flensburg and C. Peterson, Nucl. Phys. B 275 (1986) 375.

41. F. Karsch and H. W. Wyld, Phys. Rev. Lett. 55 (1985) 2242. H. Gausterer and J. R. Klauder, Phys. Lett. B 164 (1985) 127; Phys. Rev. Lett. 56 (1986) 306; Phys. Rev. D 33 (1986) 3678. E. M. Ilgenfritz, Phys. Lett. B 181 (1986) 327. J. Ambjorn and S. K. Yang, Nucl. Phys. B 275 (1986) 18. N. Bilic, H. Gausterer and S. Sanielevici, Phys. Lett. B 198 (1987) 235; Phys. Rev. D 37 (1988) 3684. C. Adami and S. E. Koonin, Phys. Rev. C 63 (2001) 034319. C. W. Bernard and V. M. Savage, Phys. Rev. D 64 (2001) 085010.

42. J. Berges, Sz. Borsanyi, D. Sexty and I.-O. Stamatescu, "Lattice simulations of realtime quantum fields", arXiv:hep-lat/0609058.

43. S. Xue, Phys. Lett. B 180 (1986) 275. 